Journal of Automata, Languages and Combinatorics $\mathbf{u}(\mathrm{v}) \mathrm{w}, \mathrm{x}-\mathrm{y}$

(c) Otto-von-Guericke-Universität Magdeburg

\title{
Tiling a Pyramidal Polycube with Dominoes
}

\author{
Olivier Bodini \\ LIRMM-UMR 5506, Université Montpellier II, \\ 161 rue Ada, 34392 Montpellier Cedex 5 - France \\ e-mail: bodini@lirmm.fr \\ and \\ DAMIEN JAMET \\ LIRMM-UMR 5506, Université Montpellier II, \\ 161 rue Ada, 34392 Montpellier Cedex 5 - France \\ e-mail: jamet@lirmm.fr
}

\begin{abstract}
The notion of pyramidal polycubes, namely the piling-up of bricks of a non-increasing size, generalizes in $\mathbb{R}^{n}$ the concept of trapezoidal polyominoes. In the present paper, we prove that $n$-dimensional dominoes can tile a pyramidal polycube if and only if the latter is balanced, that is, if the number of white cubes is equal to the number of black ones for a chessboard-like coloration, generelizing the result of [BC92] when $n=2$.

Keywords: polyomino, tiling, domino.
\end{abstract}

\section{Introduction}

The problem of domino tiling is a rather classical problem in the literature. It consists in deciding whether a polyomino (a simply-connected finite union of unit integer squares) is tilable by dominoes (a union of two edge-adjacent squares), and possibly, in computing such a tiling.

In 1990, Thurston [Thu90], using Conway's groups, introduced new important ideas which have provided a linear time algorithm to solve the problem of domino tiling. Since then, many authors have generalized these concepts. They have considered tilings with bars [KK92], domino tilings of polyominoes with holes [Thi03], enumeration on the structure of the set of tilings [DMRR04], random generation of tilings [LRS01, Wil04]. Meanwhile, Moore and Robson [MR01] and Beauquier, Nivat, Remila and Robson [BNRR95] studied the NP-completeness of several tiling problems.

In 1992, Bougé and Cosnard [BC92] proved that a trapezoidal polyomino, that is, a piling-up of rectangles of a non-increasing size, is tilable by dominoes if and only if it is balanced (that is, the number of white squares is equal to the number of black ones for a chessboard-like coloration of $\mathbb{Z}^{2}$ ). 
The aim of this paper is to extend this theorem to pyramidal polycubes, namely a natural extension of trapezoidal polyomino to any dimension.

Roughly speaking, a pyramidal polycube is a piling-up of bricks of a non-increasing size (see Figure 1).
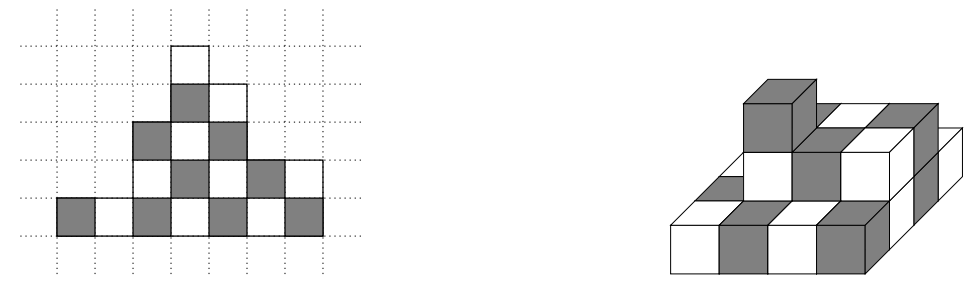

Figure 1: Left: A trapezoidal polyomino. Right: A 3-dimensional pyramidal polycube.

To prove this result, we introduce a subset of the set of pyramidal polycubes, that is, the well-unfoldable pyramidal polycubes. Roughly speaking, a pyramidal polycube $P$ is well-unfoldable if it contains a Hamiltonian path $w$ of its basis (its lower brick) allowing us to unfold $P$ as a pyramidal polyomino $P_{w}$, which is tilable if and only if so is $P$ [BC92]. Moreover, any tiling of $P_{w}$ provides in a constructive way a tiling of $P$. Finally, we show that every pyramidal polycube $P$ contains a well-unfoldable one which is tilable if and only if $P$ is tilable.

This paper is sketched as follows: in Section 1, we introduce several basic notions used in the present paper (unit cubes, polycubes, trapezoidal polyominoes and pyramidal polycubes, dominos, tilability by dominoes...) and we give a shorter proof of L. Bougé and M. Cosnard's theorem [BC92], using a sub-class of trapezoidal polyominoes, namely the regular ones. Section 2 deals with the pyramidal polycubes. In this section, we introduce the well-unfoldable pyramidal polycubes, and show that such a polycube is tilable if and only if it is balanced. Finally, we prove that, for the dominotiling problem, each pyramidal polycube can be supposed to be well-unfoldable. To do this, we consider reduced pyramidal polycubes, obtained by removing balanced bricks from the boundary of pyramidal polycubes. We conclude this section by stating the main result of the present paper, namely, that a pyramidal polycube is tilable by dominoes if and only if it is balanced.

\section{Basic notions}

The aim of this section is to introduce the basic notions we use in the sequel of this article. Firstly, we define the notions of unit cubes, polyominoes, dominoes and the notion of tilability by dominoes. Secondly, we provide the grid $\mathbb{Z}^{n}$ with a chessboardlike coloration which gives a necessary condition for a polyomino to be tilable by dominoes. Finally, we define the notion of pyramidal polycubes for any dimension $n$, and give a short proof of L. Bougé and M. Cosnard's theorem, stating that a trapezoidal polyomino $P$, namely a pyramidal polycube in dimension 2 , is tilable by dominoes if and only if it is balanced, that is, the number of white unit squares is equal to the number of black ones included in $P$. 
From now on, $n$ denotes a natural integer strictly greater than 1 .

\subsection{General notions}

Assume $\left\{\mathbf{e}_{1}, \ldots, \mathbf{e}_{\mathbf{n}}\right\}$ to be the canonical basis of the $\mathbb{R}$-vector space $\mathbb{R}^{n}$. For any vector $\mathbf{x} \in \mathbb{R}^{n}$, let us denote by $x_{i} \in \mathbb{R}$, its i-th component in the basis $\left\{\mathbf{e}_{\mathbf{1}}, \ldots, \mathbf{e}_{\mathbf{n}}\right\}$.

A point of $\mathbb{Z}^{n}$ is called a unit cube. If $n=2$, a unit cube is also called a unit square. The reason of this terminology comes from the fact that one usually represents the unit cube $\mathbf{x}$ as the cube in $\mathbb{R}^{n}$ of edge 1 and centered at $\mathbf{x}$, or with $\mathbf{x}$ as its lower vertex (i.e. with the lower coordinates). In the sequel of the present article we indifferently use both representations. For instance, the $\mathbb{Z}^{n}$-representation is more hepful for a graph theoretical approach whereas the $\mathbb{R}^{n}$-one is more helpful for a topological approach.

A polycube is a simply-connected (for the usual topology of $\mathbb{R}^{n}$ ) finite union of unit cubes. In dimension 2, a polycube is also called a polyomino.

Two unit cubes $\mathbf{x} \in \mathbb{Z}^{n}$ and $\mathbf{y} \in \mathbb{Z}^{n}$ are said to be adjacent if $\|\mathbf{x}-\mathbf{y}\|_{1}=$ $\sum_{i=1}^{n}\left|x_{i}-y_{i}\right|=1$. A union of two adjacent unit cubes is called a domino (see Figure 2).

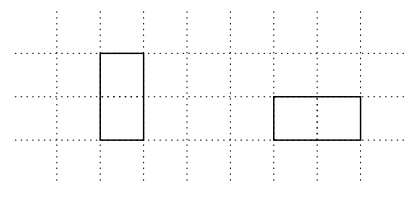

Figure 2: Left: A vertical domino. - Right: An horizontal domino.

A polycube $P$ is said to be tilable by dominoes, or just tilable for short, if $P$ is a disjoint union of dominoes (see Figure 3 ).
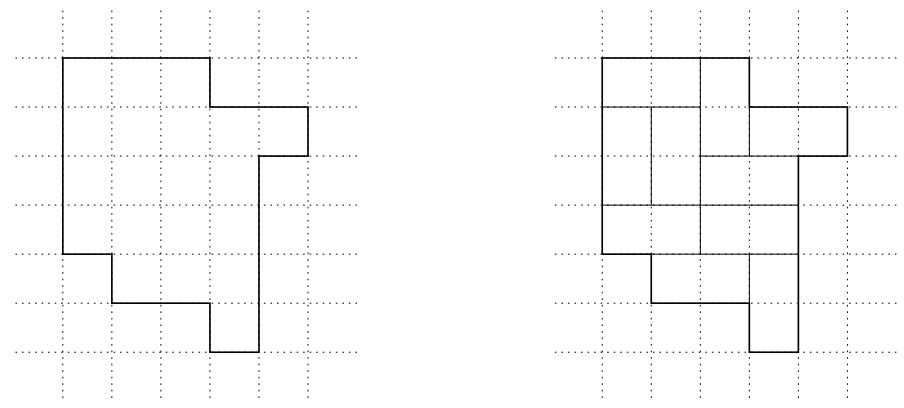

Figure 3: Left: A polyomino $P$. - Right: A domino-tiling of $P$.

Let $\mathbf{x}$ and $\mathbf{y}$ be two unit cubes and let us assume that $\{\mathbf{x}, \mathbf{y}\}$ is a domino. Then, it is clear that $\|\mathbf{x}\|_{1}$ and $\|\mathbf{y}\|_{1}$ do not have the same parity. Hence, a necessary condition for a polycube $P$ to be tilable is to include the same number of unit cubes with an even norm as the number of unit cubes with an odd norm. For this reason, we provide $\mathbb{Z}^{n}$ with a chessboard-like coloration depending on the parity of $\|\mathbf{x}\|_{1}$ : a unit cube 
$\mathbf{x} \in \mathbb{Z}^{n}$ is said to be white (resp. black) if $\|\mathbf{x}\|_{1}$ is even (resp. odd). As mentioned above, the unit cubes included in a domino are of different colors, and, if a polycube $P$ is tilable by dominoes, then the number of white cubes included in $P$ must be equal to the number of black ones. If this property holds, the polycube $P$ is said to be balanced.

Generally, the balance condition is not sufficient for a polycube to be tilable. See Figure 4 for a counter-example.

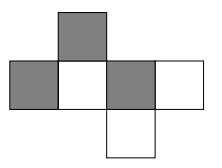

Figure 4: An untilable balanced polyomino.

In order to define the pyramidal polycubes, let us introduce the notion of brick. Let $\mathbf{x} \in \mathbb{Z}^{n}$ and $d \in \mathbb{N}^{n}$. The (unit) brick $B(\mathbf{x}, d)$ is the polycube defined as follows:

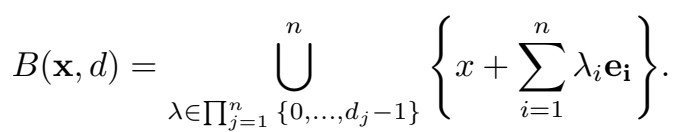

The number $d_{n}$ is called the height of the brick $B(\mathbf{x}, d)$. In dimension 2 , a brick is a usual rectangle of width $d_{1}$ and height $d_{2}$. In dimension 3 , a brick is a rectangular parallelepiped of depth $d_{1}$, width $d_{2}$ and height $d_{3}$ (see Figure 5).
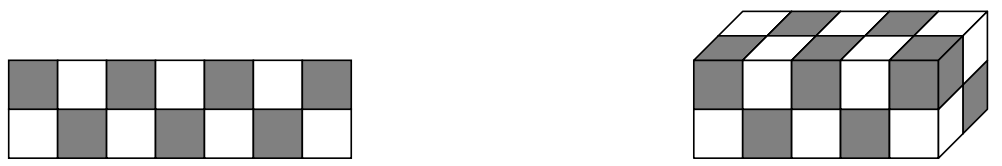

Figure 5: Left: a two-dimensional brick - Right: a three-dimensional brick.

Let us now define the notion of pyramidal polycubes. Roughly speaking, a pyramidal polycube is a piling-up of bricks of a non-increasing size along the vector $\mathbf{e}_{\mathbf{n}}$. More precisely, a polycube $P$ is said to be a pyramidal polycube, and is denoted by $P=\left(B_{1}, \ldots, B_{k}\right)$, if there exists a finite non-increasing sequence $B_{1} \supseteq B_{2} \supseteq \cdots \supseteq B_{k}$, such that:

$$
P=\bigcup_{i=1}^{k}\left(B_{i}+\sum_{j=0}^{i-1} h_{j} \mathbf{e}_{\mathbf{n}}\right),
$$

where $h_{0}=0$ and $h_{i}$ denotes the height of the brick $B_{i}$, for $i \in\{1, \ldots, k\}$. If $n=2$, a pyramidal polycube is also called a trapezoidal polyomino (see Figure 6 and Figure 7). The brick $B_{1}$ is called the basis of the pyramidal polycube $P=\left(B_{1}, \ldots, B_{k}\right)$. From now on, we only consider standard representations of pyramidal polycubes, namely the representation $\left(B_{1}, \ldots, B_{k}\right)$ where each brick is of height 1 . 

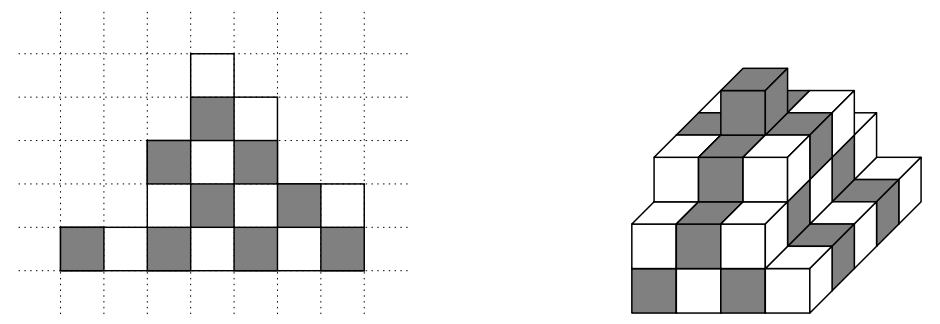

Figure 6: Left: a trapezoidal polyomino. - Right: a 3-dimensional pyramidal polycube.
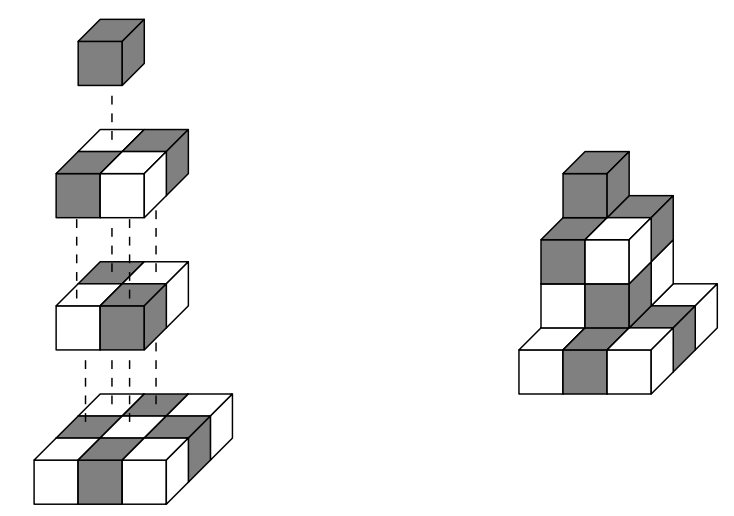

Figure 7: Left: a sequence of bricks of a non-increasing size - Right: the associated pyramidal polycube.

\subsection{Trapezoidal polyominoes}

Before proving L. Bougé and M. Cosnard's theorem, let us first introduce a notation and a terminology for the special case of trapezoidal polyominoes.

Notation. - Since, the tilability of a polyomino is invariant by an integer-vector translation, one can identify a trapezoidal polyomino $P$ with the heights $\left(h_{1}, \ldots, h_{l}\right)$ of the columns piled-up over the unit cubes of the basis (see Figure 8).

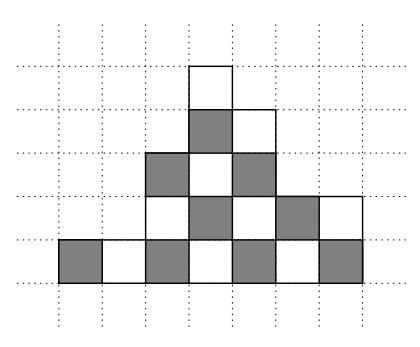

Figure 8: The trapezoidal polyomino whose height-representation is $P=(1,1,3,5,4,2,2)$. 
A particular class of trapezoidal polyominoes is the class of regular ones. A trapezoidal polyomino $P=\left(h_{1}, \ldots, h_{k}\right)$ is said to be regular if there exists $i_{0} \in\{1, \ldots, k\}$ such that $P=\left(1,2, \ldots, i_{0}-1, i_{0}, i_{0}+1, \ldots, 2,1\right)$ (see Figure 9 ). A first remark is

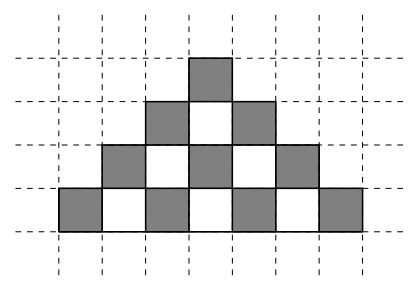

Figure 9: The regular trapezoidal polyomino whose height-representation is $P=$ $(1,2,3,4,3,2,1)$.

that a regular trapezoidal polyomino is not balanced. Indeed, the columns with even height are balanced while the ones with odd height have always an excess of the same color. We can now easily deduce that:

Theorem 1 [BC92] A trapezoidal polyomino $P$ is tilable by dominoes if and only if it is balanced.

Proof. We have already mentioned that the balance condition is necessary. Conversely, let us suppose that $P$ is balanced. Then, $P$ is not regular and, by definition contains at least one domino on its boundary (see Figure 10). By removing it from
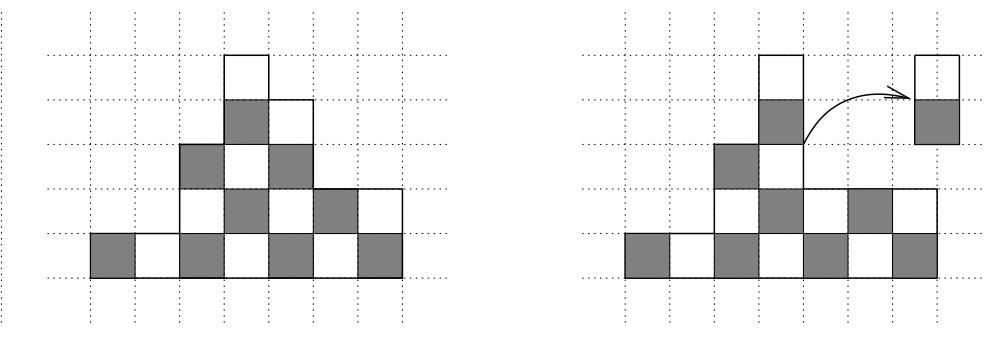

Figure 10: Tiling a non-regular balanced trapezoidal polyomino.

$P$, we obtain an other balanced trapezoidal polyomino strictly included in $P$. We conclude by induction on the number of unit cubes of $P$.

\section{The pyramidal polycubes}

In this section, we investigate the general case of pyramidal polycubes. For clarity issues, we give all the proofs for the 3-dimensional case. The forthcoming material extends in a straightforward way to any dimension.

A first intuitive idea to tile a pyramidal polycube $P$ with dominoes is to proceed by erosion, that is, by removing balanced bricks from the boundary of $P$ with the 
constraint to keep a pyramidal polycube. It is exactly what we did in the proof of Theorem 1 in the two-dimensional case. In fact, this method fails in the general case (see Figure 11): we obtain a pyramidal polycube from which we cannot remove any balanced brick from the boundary by keeping a pyramidal polycube. Such a pyramidal polycube is said to be reduced. More precisely, a reduced pyramidal polycube is a pyramidal polycube $P$, such that, for any balanced brick $B$ intersecting $P, P \backslash B$ is not a pyramidal polycube.
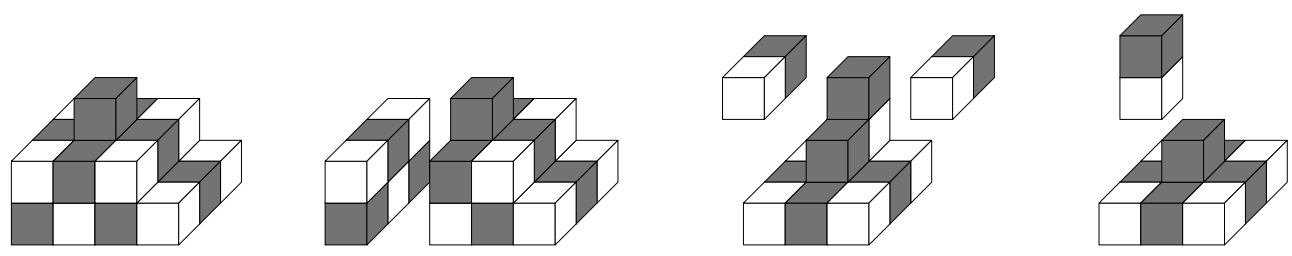

Figure 11: A tilable pyramidal polycube untilable by erosion.

A quite unexpected fact is that the $n$-dimensional tiling problem for pyramidal polycubes can be reduced to a two-dimensional tiling problem for trapezoidal polyominoes. In fact, given a pyramidal polycube $P$, after having removed all the balanced bricks we can from the boundary of $P$, we obtain a pyramidal polycube $P_{1}$, which is tilable if and only if so is $P$. Moreover, $P_{1}$ can be associated to a trapezoidal polyomino $P_{2}$ with the same balance. Finally, every tiling of $P_{2}$ provides in a canonical way a tiling of $P_{1}$, and then provides a tiling of $P$.

In this section, we first introduce the notion of well-unfoldable pyramidal polycubes and show that, such a polycube is tilable if and only if it is balanced. Next, we introduce the stratifiable pyramidal polycubes which are shown to be unfoldable. Finally, we prove that every reduced pyramidal polycube is stratifiable, and hence is tilable if and only if it is balanced. By definition of reduced pyramidal polycubes, we deduce that a pyramidal polycube is tilable if ad only if it is balanced.

\subsection{The well-unfoldable pyramidal polycubes}

The aim of this section is to show that such a pyramidal polycube includes a particular Hamiltonian path in its basis, called an Ariadne's thread, reducing the tiling problem to a tiling problem in two dimensions.

Let us begin with giving several definitions. Let $P$ be a polycube. The adjacency graph of $P$ is the bipartite graph $G_{P}$ whose vertices are the unit cubes included in $P$ partitioned by their color and whose edges are the adjacency relations between unit cubes. With this point of view, a tiling of $P$ by dominoes is equivalent to a perfect matching of $P$ (see Figure 12). Let $P$ be a pyramidal polycube. The adjacency graph of its basis is called the adjacency basis graph of $P$.

In 3 dimensions, another way to define pyramidal polycubes is to consider them as a union of columns piled-up over a plane. Let us formulate a similar point a view in the any dimension case. We denote by $\mathcal{C}(\mathbf{x}, h)$ and we call column of height $h \in \mathbb{N}^{\star}$ 

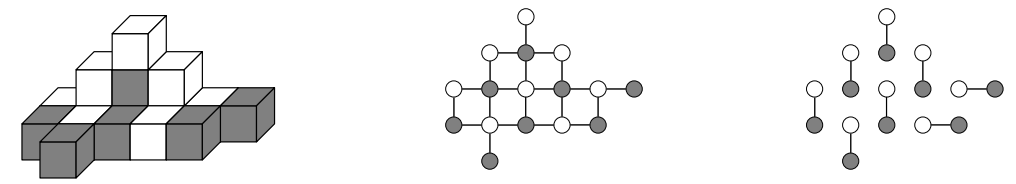

Figure 12: Left to right: a polycube - its adjacency graph - a perfect tiling.

piled-up over the unit cube $\mathbf{x} \in \mathbb{Z}^{2}$, the following polycube:

$$
\mathcal{C}(\mathbf{x}, h)=\bigcup_{i=0}^{h-1}\left\{\mathbf{x}+(i-1) \mathbf{e}_{\mathbf{n}}\right\} .
$$

Let us notice that the colum of height 1 over the unit cube $\mathbf{x}$ is nothing but $\mathbf{x}$ itself.

Hence, viewing the $n$-dimensional polycube as a union of columns piled-up over an hyperplane, we can consider a pyramidal polycube $P=\left(B_{1}, \ldots, B_{k}\right)$ as a map $\widetilde{P}: B_{1} \longrightarrow \mathbb{N}^{\star}$ mapping each unit cube of $B_{1}$ to the height of the column piled-up over it. Considering a standard representation of $P=\left(B_{1}, \ldots, B_{k}\right)$, that is, each brick is of height 1 , an explicit formula of $\widetilde{P}$ is (see Figure 13):

$$
\begin{aligned}
\widetilde{P}: B_{1} & \longrightarrow \operatorname{\mathbb {N}^{\star }} \\
\mathbf{x} & \mapsto \max \left\{j \in\{1, \ldots, k\} \mid \mathbf{x} \in B_{j}\right\} .
\end{aligned}
$$
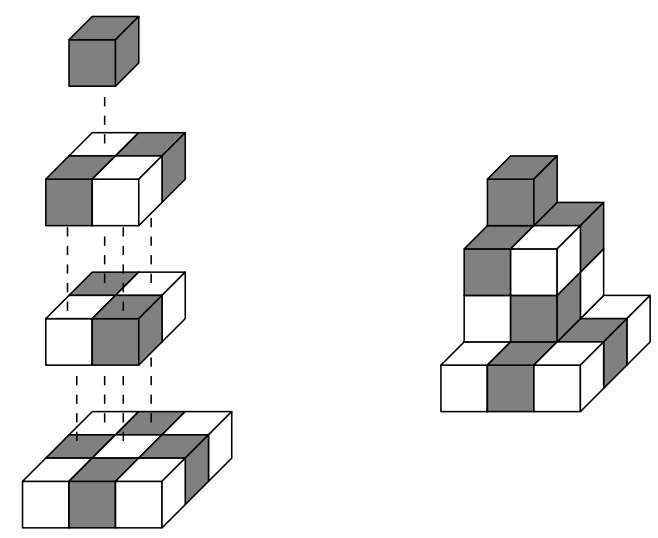

\begin{tabular}{|l|l|l|}
\hline 4 & 3 & 1 \\
\hline 3 & 3 & 1 \\
\hline 1 & 1 & 1 \\
\hline
\end{tabular}

Figure 13: Left: a sequence of bricks of non-increasing size - Center: the associated pyramidal polycube. - Right: its height-represention

Let $P=\left(B_{1}, \ldots, B_{k}\right)$ be a pyramidal polycube. Let $w=\left(w_{1}, \ldots, w_{m}\right)$ be a Hamiltonian path of the adjacency basis graph of $P$. Let $h_{i} \in \mathbb{N}^{\star}$ be the height of the columns piled-up over the unit cube $w_{i}, i \in\{1, \ldots, m\}$. Then,

$$
P=\bigcup_{i=1}^{m} \mathcal{C}\left(w_{i}, h_{i}\right)
$$


Let us consider the polyomino $P_{w}=\left(h_{1}, \ldots, h_{m}\right)$ (in a height-representation), called the $w$-unfold of $P$ (see Figure 14). Since $P_{w}$ is connected, we easily deduce that $P_{w}$
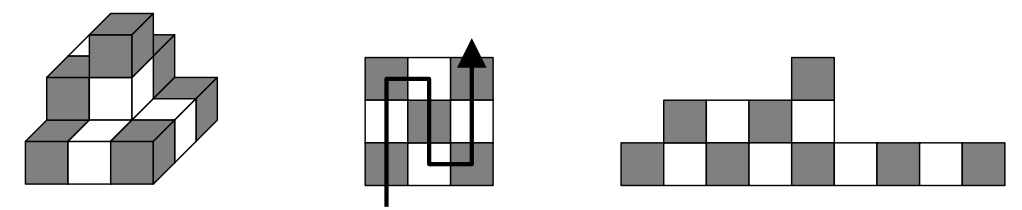

Figure 14: Left to right: a pyramidal polycube - a Hamiltionian path of its adjacency basis graph - the corresponding unfold.

and $P$ have the same balance. Moreover, a quite interesting fact is:

Proposition 2 The adjacency graph of $P_{w}$ is isomorphic to a partial graph of the adjacency graph of $P$. In particular, if $P_{w}$ is tilable then so is $P$.

Proof. Roughly speaking we want to show that unfolding a pyramidal polycube does not create any adjacency relation. Let $\Phi: P \longrightarrow P_{w}$ be the map defined by:

$$
\begin{aligned}
& \Phi: \quad P \quad \longrightarrow \quad P_{w} \\
& w_{i}+j_{i} \mathbf{e}_{\mathbf{n}} \mapsto i \mathbf{e}_{\mathbf{1}}+j_{i} \mathbf{e}_{\mathbf{2}},
\end{aligned}
$$

for $i \in\{1, \ldots, m\}$ and $j_{i} \in\left\{0, \ldots, h_{m}-1\right\}$. It is clear that $\Phi$ is one-to-one. Let $i \mathbf{e}_{1}+j \mathbf{e}_{\mathbf{2}}$ and $i^{\prime} \mathbf{e}_{\mathbf{1}}+j^{\prime} \mathbf{e}_{\mathbf{2}}$ be two unit squares of $P_{w}$ and let us assume them to be adjacent, that is, $\left|i^{\prime}-i\right|+\left|j^{\prime}-j\right|=1$.

- If $\left|i^{\prime}-i\right|=1$, then $j=j^{\prime}$. Since $w_{i}$ and $w_{i^{\prime}}$ are adjacent by definition of $w$, we deduce that $w_{i}+j \mathbf{e}_{\mathbf{n}}$ and $w_{i^{\prime}}+j^{\prime} \mathbf{e}_{\mathbf{n}}$ are adjacent too.

- If $\left|j^{\prime}-j\right|=1$, then $i=i^{\prime}$ and $w_{i}+j \mathbf{e}_{\mathbf{n}}$ and $w_{i^{\prime}}+j^{\prime} \mathbf{e}_{\mathbf{n}}$ are adjacent.

A particular class of pyramidal polycubes is the class of the ones admitting a trapezoidal unfold. Such a pyramidal polycube is said to be well-unfoldable. A Hamiltionian path of the adjacency basis graph of a pyramidal polycube $P$ providing a trapezoidal unfold is called an Ariadne's thread of $P$. A direct consequence of the previous proposition is:

Corollary 3 A well-unfoldable pyramidal polycube is tilable by dominoes if and only if it is balanced.

Proof. We have already seen that the balance condition is necessary. Conversely, let $P$ be a well-unfoldable balanced pyramidal polycube, $w$ be an Ariadne's thread of $P$ and $P_{w}$ be the $w$-unfold of $P$. Then, $P_{w}$ is balanced and is tilable from Theorem 1, and we conclude that $P$ is tilable by Proposition 2 .

Unfortunately, every pyramidal polycube is not necessarily well-unfoldable (see Figure 15). It remains now to exhibit a non-empty set of well-unfoldable pyramidal polycubes. 


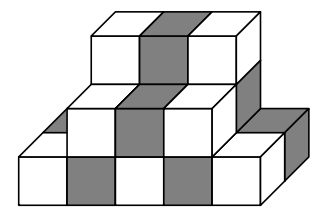

Figure 15: A non well-unfoldable pyramidal polycube.

\subsection{The stratifiable pyramidal polycubes}

Roughly speaking, a pyramidal polycube $P=\left(B_{1}, \ldots, B_{k}\right)$ is said to be stratifiable if each brick $B_{i}$ is a disjoint union of unbalanced bricks. In this section, we show that each stratifiable pyramidal polycube is well-unfoldable and then, is tilable if and only if it is balanced.

Definition 1 (Stratifiable pyramidal polycube) A pyramidal polycube $P=$ $\left(B_{1}, \ldots, B_{k}\right)$ is said to be stratifiable if there exist a finite sequence $\left(S_{1}, \ldots, S_{m}\right)$ of unbalanced bricks such that, for all $j \in\{1, \ldots, m\}, \bigcup_{i=1}^{j} S_{i}$ is a brick, and a finite non-increasing sequence $\left(m_{1}, \ldots, m_{k}\right)$ of $\{1, \ldots, m\}$ such that, for all $j \in\{1, \ldots, k\}$, $\bigcup_{i=1}^{j} S_{i}=B_{j}$.
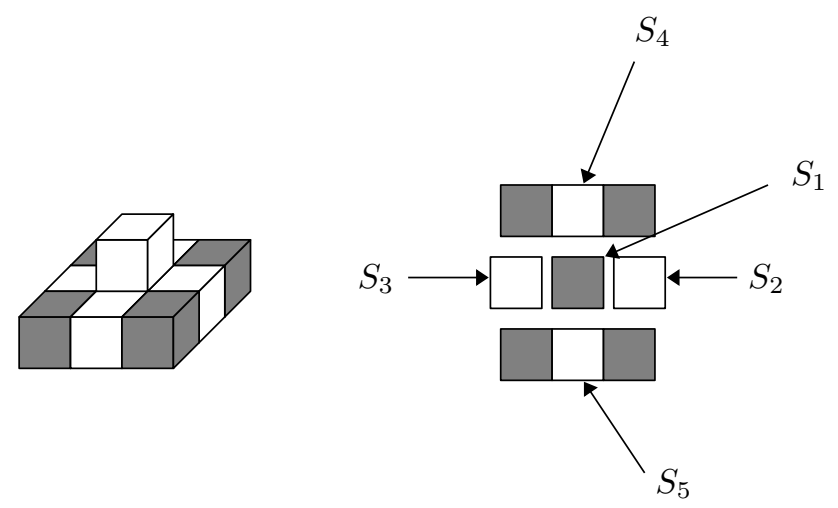

Figure 16: Left to right: a pyramidal polycube - a stratification such that $S_{1}=B_{2}$ and $S_{1} \cup S_{2} \cup S_{3} \cup S_{4} \cup S_{5}=B_{1}$.

We state now the main result of this section:

Theorem 4 A stratifiable pyramidal polycube is well-unfoldable.

Proof. Let us first notice that a brick $B(\mathbf{x}, d)$ is balanced if and only if one of the $d_{i}$ 's is even. Indeed, if all the $d_{i}$ 's are odd, then the number of unit cubes included in $B(\mathbf{x}, d)$ is odd and $B(\mathbf{x}, d)$ cannot be balanced. Conversely, let $D_{k}=\prod_{j=1}^{k}\left\{0, \ldots, d_{j}-1\right\}$, 
for $k \in\{1,2,3\}$. If one $d_{i}$ 's is even, for instance $d_{3}$, then

$$
\begin{aligned}
B(\mathbf{x}, d) & =\bigcup_{\lambda \in D_{3}}\left\{\mathbf{x}+\lambda_{1} \mathbf{e}_{\mathbf{1}}+\lambda_{2} \mathbf{e}_{\mathbf{2}}+\lambda_{3} \mathbf{e}_{\mathbf{3}}\right\} \\
& =\bigcup_{\lambda \in D_{2}} \bigcup_{\lambda_{3}=0}^{d_{3}-1}\left\{\mathbf{x}+\left(\lambda_{1} \mathbf{e}_{\mathbf{1}}+\lambda_{2} \mathbf{e}_{\mathbf{2}}\right)+\lambda_{3} \mathbf{e}_{\mathbf{3}}\right\} .
\end{aligned}
$$

Moreover, for any 2-uple $\lambda \in D_{2}$, the polycube $\bigcup_{\lambda_{3}=0}^{d_{3}-1}\left\{\mathbf{x}+\left(\lambda_{1} \mathbf{e}_{\mathbf{1}}+\lambda_{2} \mathbf{e}_{\mathbf{2}}\right)+\lambda_{3} \mathbf{e}_{\mathbf{3}}\right\}$ is balanced since $d_{3}$ is even and the result follows.

Secondly, by a similar decomposition of a brick, we deduce that a balanced (resp. unbalanced) brick admits a Hamiltonian path linking any couple of endpoints of an even edge (resp. linking two diametrically opposite unit cubes).

Let $\left(S_{1}, \ldots S_{m}\right)$ be a stratification of $P$ and let $\left(m_{1}, \ldots, m_{k}\right)$ be the corresponding non-increasing sequence. Let $T_{j}=\bigcup_{i=1}^{j} S_{i}$. Then, for all $j \in\{1, \ldots, k\}, T_{m_{k}}=B_{k}$ and $T_{j}$ is balanced if and only if $j$ is even.

- Assume that $m_{k}$ is even. Then $B_{k}$ admits a Hamiltonian path linking two endpoints of an even edge of $B_{k}$. Since $S_{m_{k}+1}$ is unbalanced, it is adjacent to $B_{k}$ by an odd edge (see Figure 17). Then, we extend the Hamiltonian path of $B_{k}$
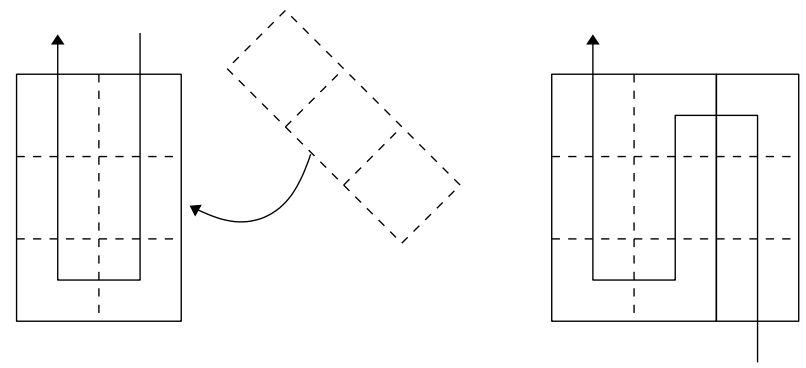

Figure 17: Left to right: adjoining an unbalanced brick to a balanced one.

to an Ariadne's thread of $\left(B_{k}, B_{k} \cup S_{m_{k}+1}\right)$ linking two diametrically opposite unit cubes of $B_{k} \cup S_{m_{k}+1}$.

- If $m_{k}$ is odd, then $B_{k}$ admits a Hamiltonian path linking two diametrically opposite unit cubes of $B_{k}$ and, whatever the common edge between $S_{m_{k}+1}$ and $B_{k}$, we can extend the Hamiltonian path of $B_{k}$ to an Ariadne's thread of $\left(B_{k}, B_{k} \cup S_{m_{k}+1}\right)$ linking the endpoints of one of its even edges (see Figure 18).

We conclude by iteration on $m$.

Unfortunately, every pyramidal polycube is not stratifiable (see Figure 19). 

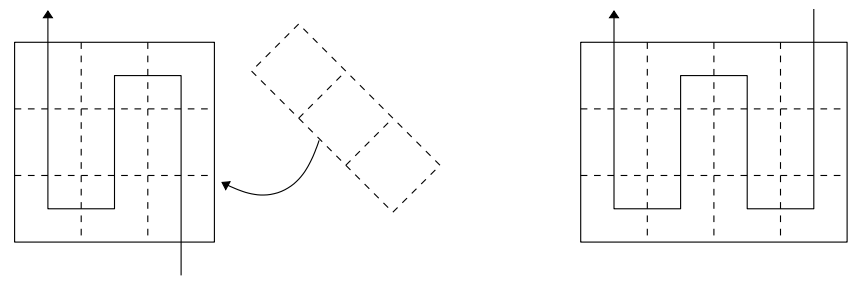

Figure 18: Left to right: adjoining two unbalanced bricks.

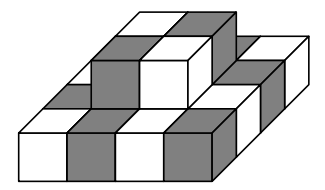

Figure 19: An unstratifiable pyramidal polycube.

\subsection{The reduced pyramidal polycubes}

In the present section we show that every pyramidal polycube contains a stratifiable pyramidal polycube, also called reduced pyramidal polycube. The main significance of a reduced pyramidal polycubes is that it is tilable by dominoes if and only if the original pyramidal polycube from which it is computed is tilable by dominoes.

Let us now recall the notion of reduced pyramidal polycube:

Definition 2 (Reduced pyramidal polycube) A pyramidal polycube $P$ is said to be reduced if for any balanced brick $B$ intersecting $P, P \backslash B$ is not a pyramidal polycube.

Let us now prove the main result of this section:

Theorem 5 A reduced pyramidal polycube is stratifiable.

Proof. Similarly as before, we only treat the three-dimensional case. Let $P=$ $\left(B_{1}, \ldots, B_{k}\right)$ be a reduced pyramidal polycube. If $k=1$, then $P$ is clearly stratifiable. Let us suppose that any reduced pyramidal polycube with height $k-1$ is stratifiable. Since $P=\left(B_{1}, \ldots, B_{k}\right)$ is supposed to be reduced, then so is $P^{\prime}=\left(R_{2}, \ldots, R_{k}\right)$, and hence, $P^{\prime}$ is stratifiable.Several cases can occur depending on $B_{1}$ and $B_{2}$. First let us notice that $B_{1}$ has at most one even edge (see Figure 20). We deduce that the configurations of $B_{2}$ in $B_{1}$ can only be the following ones (see Figure 21), and we see in each case how to deduce a stratification of $P$ from a stratification of $\left(B_{2}, \ldots, B_{k}\right)$. Indeed, it is sufficient to see that $B_{1}$ is a union of $B_{2}$ and unbalanced brick $\left(S_{1}, \ldots, S_{m}\right)$ with $B_{2} \cup S_{1} \cup \cdots \cup S_{j}$, for all $j \leq m$ is a brick, which is immediate (see Figure 21).

A consequence of this theorem is: 

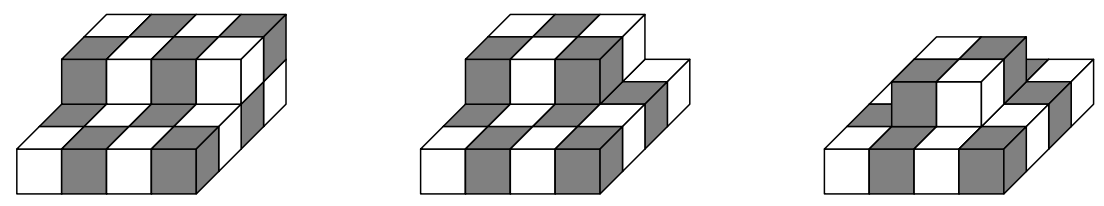

Figure 20: If $B_{1}$ has two even edges, then $P$ is not reduced.
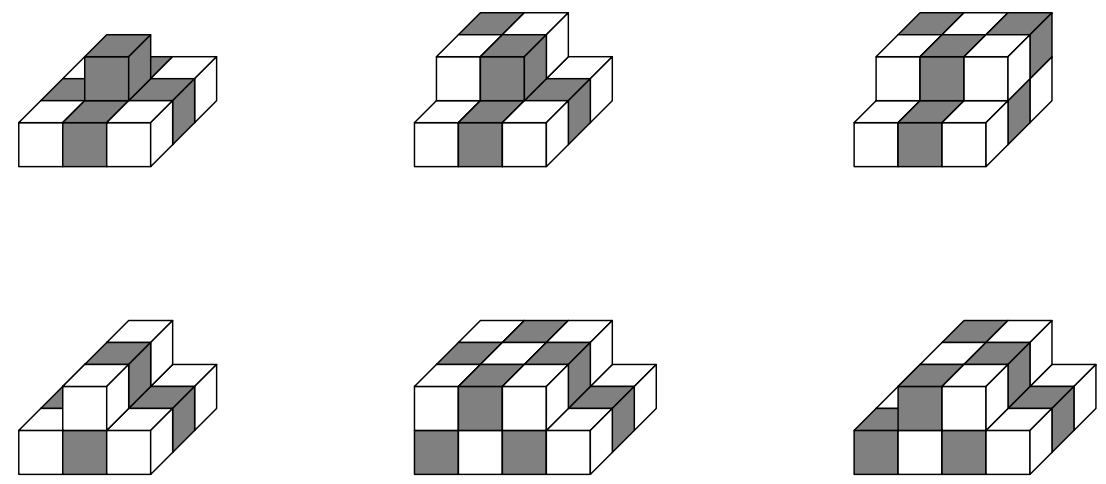

Figure 21: The different possible configurations for $B_{1}$ and $B_{2}$.

Corollary 6 Let $P$ be a pyramidal polycube and $P^{\prime}$ be a reduced pyramidal polycube of $P$, computed by removing balanced bricks from the boundary of $P$. Then $P$ is tilable by dominoes if and only if so is $P^{\prime}$.

Proof. By construction, it is clear that $P$ is tilable if so is $P^{\prime}$. Conversely, let us suppose that $P$ is tilable. Then $P$ is balanced and $P^{\prime}$ too. From Theorem 5, we deduce that $P^{\prime}$ is well-unfoldable and by Corollary 3 , we conclude that $P^{\prime}$ is tilable by dominoes.

We can now state the main result of this paper:

Theorem 7 A pyramidal polycube is tilable by dominoes if and only if it is balanced.

Proof. The balance condition is clearly necessary. Conversely, assume $P$ to be a balanced pyramidal polycube and $P^{\prime}$ be a reduced pyramidal polycube computed from $P$ by removing balanced bricks from the boundary of $P$. Then, $P^{\prime}$ is balanced and is tilable by dominoes by Theorems 5 and 4 and Corollary 3 . We conclude with Corollary 6.

\section{Conclusion}

In the present paper, we have generalized to any dimension a result due to L. Bougé and $\mathrm{M}$. Cosnard [BC92], stating that a trapezoidal polyomino is tilable by dominoes if and only if it is balanced, considering the pyramidal polycubes as a natural extension 
of the trapezoidal polyominoes. A first track to be investigated could be to exhibit a shorter proof of this result as done for the two-dimensional case. Secondly, it could be interested to generalize this kind approach (using a particular Hamiltionian path) to more general tiles (bars of length 3 , rectangles...).

\section{References}

[BC92] L. Bougé and M. Cosnard. Tiling a trapezoidal region with dominoes. C.R. Acad. Sci. Paris Sér. I Math, 315(2):221-226, 1992.

[BNRR95] D. Beauquier, M. Nivat, É. Rémila, and M. Robson. Tiling figures of the plane with two bars. Comput. Geom., 5:1-25, 1995.

[DMRR04] S. Desreux, M. Matamala, I. Rapaport, and É. Rémila. Domino tilings and related models: space of configurations of domains with holes. Theor. Comput. Sci., 319(1-3):83-101, 2004.

[KK92] C. Kenyon and R. Kenyon. Tiling a polygon with rectangles. In IEEE Symposium on Foundations of Computer Science, pages 610-619, 1992.

[LRS01] M. Luby, D. Randall, and A. Sinclair. Markov chain algorithms for planar lattice structures. SIAM J. Comput., 31(1):167-192, 2001.

[MR01] C. Moore and J.M. Robson. Hard tiling problems with simple tiles. Discrete \&3 Computational Geometry, 26(4):573-590, 2001.

[Thi03] N. Thiant. An o(n $\log \mathrm{n})$-algorithm for finding a domino tiling of a plane picture whose number of holes is bounded. Theor. Comput. Sci., 2-3(303):353-374, 2003.

[Thu90] William P. Thurston. Conway's tiling groups. Am. Math. Monthly, 97(8):757-756, 1990.

[Wil04] D.B. Wilson. Mixing times of lozenge tiling and card shuffling markov chains. Ann. Appl. Probab., 14(1):274-325, 2004. 